\title{
Histologic discrepancy between endoscopic forceps biopsy and endoscopic mucosal resection specimens of colorectal polyp in actual clinical practice
}

\author{
Moon Joo Hwang, Kyeong Ok Kim, A Lim Kim, Si Hyung Lee, Byung Ik Jang, Tae Nyeun Kim \\ Division of Gastroenterology and Hepatology, Department of Internal Medicine, Yeungnam University College of Medicine, Daegu, Korea
}

\begin{abstract}
Background/Aims: We aimed to assess the rate of histologic discrepancy (HD) between endoscopic forceps biopsy (EFB) and totally resected specimens in colorectal polyp and analyze the risk factors of discordant group, especially under-diagnosis (UD) cases before complete removal of colorectal polyp. Methods: From 2010 to 2015, a total of 290 polyps in 210 patients which had baseline pathology report before endoscopic resection (ER) were analyzed. UD cases were defined as those in which the diagnosis changed to a more advanced histologic feature after ER. Results: A change in the final histology after ER was noted in 137 cases (47.2\%), and after excluding 9 insignificant cases, 128 cases were further categorized into overdiagnosed and under-diagnosed group. UD occurred in 86 cases $(29.7 \%)$ and change from benign to malignancy was noted in 26 cases $(8.9 \%)$. On univariate analysis, a larger polyp size $(>10 \mathrm{~mm})$ was significantly associated with both $\mathrm{HD}(P<0.001)$ and UD $(P<0.001)$. Regarding polyp morphology, protruding or flat was not significantly important. On multivariate analysis, polyp size $>10 \mathrm{~mm}$ was the single most significant predictor of both HD $(P<0.001)$ and UD $(P<0.001)$. Conclusions: The HD and UD rates were $47.2 \%$ and $29.7 \%$, respectively. Polyp size $>10 \mathrm{~mm}$ was the most important predictor of both HD and UD. We should be careful in making treatment strategy of colorectal polyp based on histologic report of EFB especially when the size of polyp is $>10 \mathrm{~mm}$. (Intest Res 2018;16:475-483)
\end{abstract}

Key Words: Colorectal polyp; Histology; Discrepancy; Biopsy; Endoscopic mucosal resection

\section{INTRODUCTION}

Colorectal cancer (CRC) is the second most common malignancy in women and third most common malignancy in men worldwide. ${ }^{1,2}$ Since tubular adenoma is recognized as precursor lesion of CRC by adenoma-carcinoma sequence, ${ }^{3}$ regular colonoscopy with excision of adenomatous polyps is associated with a significantly reduced incidence of CRC. ${ }^{4,5}$ Endoscopic resection (ER) is an effective treatment option for colon neoplasm including adenoma, carcinoma in situ,

Received January 23, 2018. Revised March 3, 2018.

Accepted March 7, 2018.

Correspondence to Kyeong Ok Kim, Division of Gastroenterology and

Hepatology, Department of Internal Medicine, Yeungnam University College of Medicine, 170 Hyeonchung-ro, Nam-gu, Daegu 42415, Korea. Tel: +82-

53-620-3830, Fax: +82-53-654-8386, E-mail: cello7727@naver.com and cancer with minimal submucosal invasion. However, ER of the polyp is not always possible at the initial colonoscopy. In actual practice, ER is not widely performed at primary medical centers owing to the risk of complications such as bleeding and perforation. In addition, the patients taking antiplatelet or anticoagulant drugs at the time of procedure are not suitable for ER. In such cases, endoscopic forceps biopsy (EFB) is usually performed in a portion of the polyp, and the patient is referred to a more specialized center. However, EFB prior to ER may sometimes result in submucosal fibrosis, making lifting of the lesion with submucosal injection difficult. $^{6}$

Although recent advances in endoscopic techniques such as contrast-enhancement techniques, whether dye-based or computed, optical microscopy, autofluorescence imaging, and narrow-band imaging would help distinguish clinically

\footnotetext{
๑ Copyright 2018. Korean Association for the Study of Intestinal Diseases. All rights reserved.

This is an Open Access article distributed under the terms of the Creative Commons Attribution Non-Commercial License (http://creativecommons.org/licenses/by-nc/4.0)

which permits unrestricted non-commercial use, distribution, and reproduction in any medium, provided the original work is properly cited.
} 
significant lesions, ${ }^{7}$ these techniques are not widely available in real clinical practice yet. Determination of histologic subtype of colorectal neoplasm by EFB is still an important step before deciding the treatment strategy of ER, surgical resection, or close observation. However, since only a small portion of the polyp is obtained with EFB, there is a risk of missing the advanced lesions or neoplastic foci within the lesion. ${ }^{8}$ There have been many studies about the histologic discrepancy (HD) between EFB and ER specimens in patients with gastric neoplasm. ${ }^{8-13}$ However, only few studies have evaluated HD in patients with colorectal neoplasm. ${ }^{8,14-18}$

The aim of this study was to evaluate the validity of a cold biopsy sample as representative for the whole resected specimen, investigate the rate of HD in colorectal neoplasm, evaluate the clinicopathological characteristics of discordant cases, and analyze the risk factors of under-diagnosis (UD) before complete removal of colorectal polyp by ER.

\section{METHODS}

\section{Patients}

We retrospectively reviewed the medical records and endoscopic findings of patients who underwent ER of colorectal polyp from January 2010 to March 2015 at Yeungnam University Medical Center. Among 1,171 patients who underwent ER of colorectal polyp, patients without biopsy results prior to ER were excluded. Patients with known synchronous adenocarcinoma, familial adenomatous polyposis, or IBD were also excluded. Moreover, patients with a nonepithelial origin neoplasm such as a neuroendocrine tumor, which usually presents as a subepithelial lesion, were excluded from this study. Consequently, a total of 290 polyps from 210 patients were included in the final analysis. The baseline characteristics of the patients, time interval between initial colonoscopy and ER, endoscopic details of the polyp including size, morphology, and histology were assessed.

The indications for ER at our hospital are in accordance with the American Society for Gastrointestinal Endoscopy guideline. ${ }^{19}$ For elderly patients who are not eligible for surgical therapy because of poor general condition, or patients who refuse to undergo surgery, ER was selectively performed.

Colonoscopy was performed after bowel preparation with polyethylene glycol electrolyte lavage solution. The size of the lesion was estimated using closed $(2 \mathrm{~mm})$ or open (7 $\mathrm{mm}$ ) biopsy forceps held against the polyp. After complete removal of the lesion, the precise size of the resected speci- men was measured with a ruler. Polyp morphology was described as protruding (Paris classification I-pedunculated or sessile) or flat (Paris classification II) subtypes, and by definition, flat lesions larger than $10 \mathrm{~mm}$ in diameter that grow laterally rather than vertically were defined as lateral spreading tumors (LSTs).$^{20}$ ER was most commonly performed using endoscopic mucosal resection (EMR), and occasionally, simple polypectomy, EMR with precutting method, or endoscopic submucosal dissection. EMR was performed with injection of epinephrine mixed with saline $(1: 10,000)$ into the submucosal space under the lesion creating a safety cushion. Then the lifted lesion was excised with a loop snare using an electrosurgical unit.

Only 5 cases of EFB histologic reports were made at our hospital, while the rest of the cases were analyzed at each local medical center. After ER was performed, totally resected specimens were analyzed by 2 pathologists who were experts in gastroenterology.

The lesions from the enrolled patients were categorized into 2 groups, the concordant and discordant groups, based on the HD of EFB and ER specimen results. In the discordant group, lesions were subsequently categorized into the overdiagnosis (OD) and UD group. The UD group was defined as that in which the diagnosis changed from benign to malignancy, low-grade dysplasia to high-grade dysplasia, less villous to more villous component, or nonneoplastic polyp to neoplastic polyp. The OD group was defined and categorized opposite to the changes in the UD group.

\section{Statistical Analysis}

Logistic regression analysis was used to determine which variable contributed to the HD, especially UD between EFB and ER specimens. A univariate analysis was carried out initially for each variable, and subsequently all variables were included in a multivariate logistic regression analysis. Each variable was analyzed with the chi-square and Student- $t$ test. Statistical significance was set at $P<0.05$. All statistical analyses were performed using the Statistical Package for Social Sciences, IBM SPSS version 20.0 (IBM Corp., Armonk, NY, USA).

\section{Ethical Considerations}

This study was approved by the Institutional Review Board of Yeungnam University Medical Center of Korea (IRB No. 2016-06-059), which exempted the requirement for informed consent because we accessed only de-identified 
data retrospectively.

\section{RESULTS}

\section{Baseline Characteristics and Histologic Results}

The mean age was $60.2 \pm 10.2$ years (range, $24-85$ years),

Table 1. Baseline Characteristics of Patients and Polyps

\begin{tabular}{lc}
\hline \multicolumn{1}{c}{ Characteristic } & Value ( $\mathbf{n = 2 1 0 )}$ \\
\hline Sex (male:female) & $143: 67(2.14: 1)$ \\
\hline Age $(\mathrm{yr})$ & $60.23 \pm 10.22$ \\
\hline BMI $\left(\mathrm{kg} / \mathrm{m}^{2}\right)$ & $23.36 \pm 3.03$ \\
\hline Comorbidities & $71(33.8)$ \\
\hline Hypertension & $56(26.6)$ \\
\hline Diabetes mellitus & $27(12.8)$ \\
\hline History of colon operation & $7(3.3)$ \\
\hline Time interval between EFB and ER (day) & $34.45 \pm 15.73$ \\
\hline Location of polyp (right/left) & $137(47.2) / 153(52.8)$ \\
\hline Cecum & $21(7.2)$ \\
\hline Ascending colon & $83(28.6)$ \\
\hline Transverse colon & $33(11.4)$ \\
\hline Descending colon & $12(4.1)$ \\
\hline Sigmoid colon & $87(30.0)$ \\
\hline Rectum & $54(18.6)$ \\
\hline Size of polyp (mm) & $17.83 \pm 9.47$ \\
\hline Morphology of polyp & \\
\hline Protruding type & $158(54.5)$ \\
\hline Flat type & $132(45.5)$ \\
\hline Lateral spreading tumor & $96(33.1)$ \\
\hline Homogenous granular & $31(32.3)$ \\
\hline Mixed nodular & $22(22.9)$ \\
\hline Flat elevated & $32(33.3)$ \\
\hline Pseudodepressed & $11(11.5)$ \\
\hline Simple polypectomy & $3(1.0)$ \\
\hline EMR & $269(92.8)$ \\
\hline EMR with precutting method & $10(3.4)$ \\
\hline Endoscopic submucosal dissection & $8(2.8)$ \\
\hline
\end{tabular}

Values are presented as mean \pm SD or number $(\%)$.

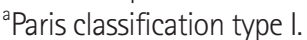

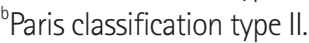

'Flat morphology larger than $10 \mathrm{~mm}$ in diameter.

$E F B$, endoscopic forceps biopsy; ER, endoscopic resection; EMR, endoscopic mucosal resection. and the male to female ratio was 2.14:1. A total of 290 polyps in 210 patients were analyzed. Among 198 cases that were stated with definite number of EFB performed, $96.5 \%$ of forceps biopsies were performed only once per colorectal polyp, probably due to concerns of massive fibrosis that might interfere with complete resection. One hundred thirty-seven (47.2\%) and 153 (52.8\%) polyps were noted in the right and left colon, respectively. The mean polyp size was $17.8 \pm 9.5$ $\mathrm{mm}$ (range, 5-50 mm). Regarding polyp morphology, 158 (54.5\%) and 132 (45.5\%) polyps were protruding and flat subtypes, respectively; among flat subtypes, 96 were LSTs (33.1\% overall) (Table 1). The most common histology with EFB was tubular adenoma low-grade dysplasia (185 cases, $63.8 \%)$.

A change in the final histology after ER was noted in 137 cases (47.2\%) (Table 2). Among 137 discordant cases, in 9 cases, it was impossible to determine whether OD or UD had occurred before ER because, for example, a change from hyperplastic polyp to chronic nonspecific inflammation or vice versa was found. Therefore, after excluding these 9 cases, a total of 128 cases were further categorized into the OD or UD group. UD occurred in 86 cases $(67.2 \%$ among HD cases; $29.7 \%$ of the overall cases) and OD occurred in 42 cases (32.8\% among HD cases; $14.5 \%$ of the overall cases). Comparisons of baseline characteristics of the UD group and the rest are summarized in Table 3. In the UD group, change from benign to malignancy was noted in 26 cases (30.2\% among UD cases, $8.9 \%$ of overall cases); among them, change from high-grade dysplasia to well-differentiated adenocarcinoma was most common $(\mathrm{n}=10,38.5 \%)$. In the OD

Table 2. Change in Histologic Results between Endoscopic Forceps Biopsy and Resected Specimens

\begin{tabular}{lc}
\hline \multicolumn{1}{c}{ Histologic results } & $\mathbf{n = 2 9 0}$ \\
\hline Discordant rate & $137(47.2)$ \\
Under-diagnosis & $86(67.2)$ \\
Benign $\rightarrow$ malignancy & 26 \\
Low grade dysplasia $\rightarrow$ high grade dysplasia & 22 \\
Change to more villous component group & 27 \\
Nonneoplastic polyp $\rightarrow$ neoplastic polyp & 11 \\
Over-diagnosis & $42(32.8)$ \\
Adenocarcinoma $\rightarrow$ high grade dysplasia & 1 \\
High grade dysplasia $\rightarrow$ low grade dysplasia & 14 \\
Change to less villous component group & 19 \\
Neoplastic polyp $\rightarrow$ nonneoplastic polyp & 8 \\
\hline
\end{tabular}

values are presented as number (\%). 
Table 3. Characteristics of Patients and Polyps in the Under-Diagnosis Group

\begin{tabular}{|c|c|c|c|}
\hline Characteristic & Under-diagnosis & Rest of others & $P$-value \\
\hline \multicolumn{4}{|l|}{ Patients } \\
\hline Sex (male:female) & 54:32 & $127: 68$ & 0.706 \\
\hline Age (yr) & $61.17 \pm 10.38$ & $60.10 \pm 10.22$ & 0.421 \\
\hline $\mathrm{BMI}\left(\mathrm{kg} / \mathrm{m}^{2}\right)$ & $21.93 \pm 3.24$ & $24.14 \pm 13.74$ & 0.853 \\
\hline Comorbidities (\%) & $25(29.1)$ & $46(37.1)$ & 0.227 \\
\hline Mean interval between initial colonoscopy and ER (day) & $30.96 \pm 25.67$ & $32.65 \pm 322.48$ & 0.670 \\
\hline \multicolumn{4}{|l|}{ Polyps } \\
\hline Location of polyp (right/left) & $35(40.7) / 51(59.3)$ & $96(49.2) / 99(50.8)$ & 0.186 \\
\hline Cecum & $5(5.8)$ & $15(7.7)$ & 0.573 \\
\hline Ascending colon & $25(29.1)$ & $53(27.2)$ & 0.744 \\
\hline Transverse colon & $5(5.8)$ & $28(14.4)$ & 0.040 \\
\hline Descending colon & $1(1.2)$ & $11(5.6)$ & 0.113 \\
\hline Sigmoid colon & $32(37.2)$ & $55(28.2)$ & 0.132 \\
\hline Rectum & $18(20.9)$ & $33(16.9)$ & 0.422 \\
\hline Size of polyp (mm) & $22.15 \pm 9.42$ & $16.21 \pm 8.99$ & $<0.001$ \\
\hline \multicolumn{4}{|l|}{ Morphology of polyp } \\
\hline Protruding type/flat type & $51(59.3) / 35(40.7)$ & $101(51.8) / 94(48.2)$ & 0.244 \\
\hline Lateral spreading tumor & $31(36.0)$ & $63(32.3)$ & 0.540 \\
\hline Homogenous granular type & $7(22.6)$ & $22(34.9)$ & \\
\hline Mixed nodular type & $10(32.3)$ & $12(19.0)$ & \\
\hline Flat elevated type & $10(32.3)$ & $22(34.9)$ & \\
\hline Pseudodepressed type & $4(12.8)$ & $7(11.2)$ & \\
\hline
\end{tabular}

Values are presented as mean \pm SD or number (\%).

$E R$, endoscopic resection.

group, a single case of change from malignancy to benign was noted. In this case, an $18 \mathrm{~mm}$ polyp was found and classified as well-differentiated adenocarcinoma in situ by EFB. At the time of ER, the polyp size was decreased to approximately $12 \mathrm{~mm}$, and the resected specimen revealed tubular adenoma with high-grade dysplasia. When we retrospectively reviewed the EFB specimen of this case at our hospital after complete resection, it came out as well-differentiated adenocarcinoma again.

\section{Factors Associated with HD, UD and OD}

The univariate analysis of clinicopathological characteristics of the HD group and UD group showed that larger $(>10$ $\mathrm{mm}$ ) polyp size (HD, $P<0.001$; UD, $P<0.001)$ and polyp location at the transverse colon (HD, $P=0.001$; UD, $P=0.040$ ) were significantly associated with both HD and UD (Table 4). Polyps located at transverse colon showed a significantly low risk of HD (OR, $0.263 ; 95 \% \mathrm{CI}, 0.110-0.628 ; P=0.001)$ and UD (OR, 0.368; 95\% CI, 0.137-0.989; $P=0.040$ ), and when comparing rectum with transverse colon, polyps at rectum had approximately 5 times greater risk of HD (OR, 5.006; 95\% CI, 1.853-13.532; $P=0.001$ ). However, when the polyp location was categorized into right and left colon divided by the splenic flexure, there was no significant association with HD and UD (HD: OR, 1.375; 95\% CI, 0.86-2.18; $P=0.178$ and UD: OR, 1.413; 95\% CI, 0.84-2.32; $P=0.186$ ). Polyp morphology whether protruding or flat was not significantly associated with HD $(P=0.206)$ or UD $(P=0.244)$. If LST was excluded among the flat subtypes, protruding morphology had an increasing tendency for both HD (OR, 4.249; 95\% CI, 1.758$10.268 ; P=0.001)$ and UD (OR, 3.913; 95\% CI, 1.310-11.690; $P=0.010)$ compared with flat morphology.

Gender, age, BMI, or time interval between EFB and ER were not significant predictors of HD or UD. On multivariate analysis, size $>10 \mathrm{~mm}$ was the only independent predictor of 
Table 4. Factors Associated with Histologic Discrepancy and Under-Diagnosis by Univariate Analysis

\begin{tabular}{|c|c|c|c|c|}
\hline Characteristic & \multicolumn{2}{|c|}{ Histologic discordancy } & \multicolumn{2}{|c|}{ Under-diagnosis } \\
\hline Sex & $1.632(0.923-2.712)$ & 0.058 & $1.106(0.653-1.876)$ & 0.706 \\
\hline Age (yr) & $1.003(0.981-1.026)$ & 0.772 & $1.008(0.985-1.032)$ & 0.492 \\
\hline BMI $\left(\mathrm{kg} / \mathrm{m}^{2}\right)$ & 1.002 (0.971-1.011) & 0.320 & $1.005(0.912-1.055)$ & 0.562 \\
\hline Interval between EFB and ER (day) & $0.998(0.991-1.006)$ & 0.661 & 0.997 (0.989-1.005) & 0.453 \\
\hline Cecum & $0.826(0.337-2.026)$ & 0.676 & $0.741(0.260-2.107)$ & 0.573 \\
\hline Ascending colon & $1.292(0.776-2.152)$ & 0.324 & $1.098(0.626-1.927)$ & 0.744 \\
\hline Transverse colon & $0.263(0.110-0.628)$ & 0.001 & $0.368(0.137-0.989)$ & 0.040 \\
\hline Descending colon & $0.358(0.095-1.351)$ & 0.115 & $0.197(0.025-1.549)$ & 0.113 \\
\hline \multicolumn{5}{|l|}{ Polyp morphology } \\
\hline Protruding vs. flat & $1.350(0.848-2.148)$ & 0.206 & $1.356(0.811-2.267)$ & 0.244 \\
\hline
\end{tabular}

EFB, endoscopic forceps biopsy; ER, endoscopic resection.

Table 5. Risk Factors of Histologic Discrepancy and Under-Diagnosis by Multivariate Analysis

\begin{tabular}{|c|c|c|c|c|}
\hline \multirow{2}{*}{ Risk factor } & \multicolumn{2}{|c|}{ Histologic discordancy } & \multicolumn{2}{|c|}{ Under-diagnosis } \\
\hline & Adjusted OR (95\% Cl) & $P$-value & Adjusted OR (95\% Cl) & $P$-value \\
\hline Size $>10 \mathrm{~mm}$ & $4.852(2.615-9.002)$ & $<0.001$ & $5.102(2.421-10.752)$ & $<0.001$ \\
\hline Locate at transverse colon & $0.451(0.111-1.833)$ & 0.266 & $0.963(0.231-4.016)$ & 0.959 \\
\hline Protruding type vs. flat type ${ }^{a}$ & $1.839(0.686-4.934)$ & 0.226 & $1.543(0.443-5.376)$ & 0.496 \\
\hline
\end{tabular}

${ }^{\mathrm{a}}$ Flat type excluding lateral spreading tumor.

both HD (OR, 4.852; 95\% CI, 2.615-9.002; $P<0.001)$ and UD (OR, 5.102; 95\% CI, 2.421-10.752; $P<0.001$ ) (Table 5).

In a subgroup analysis of polyp morphology according to polyp size $>10 \mathrm{~mm}$ or $\leq 10 \mathrm{~mm}$, polyp morphology was not a significant risk factor for HD in both circumstances. Although LST itself was not significantly associated with HD $(P=0.245)$ or UD $(P=0.540)$, each of the 4 subtypes were compared within LST. Among the 4 LST subtypes, mixed nodular type was significantly associated with HD (OR, 5.906; 95\% CI, 1.820-19.163; $P=0.001$ ). However, none of the LST subtypes were significantly associated with UD (Table 6).

In the subgroup analysis of predictive factors of change from benign to malignancy ( $n=26$ ), which is clinically the most significant pitfall, size was the most important predictive factor. The mean size of such cases was $23.42 \pm 8.33 \mathrm{~mm}$, while the mean size of the rest was $17.47 \pm 9.74 \mathrm{~mm}(P<0.001)$. In addition, polyps located in the left colon had a higher ten- dency to eventually be identified as malignancy (OR, 2.571; 95\% CI, 1.044-6.329; $P=0.035$ ). On the other hand, polyp morphology was not significantly associated with change from benign to malignancy $(P=0.539)$.

In comparison between OD group and the rest of others, size again revealed to be a significant predictor of OD (OD group $23.9 \pm 10.4 \mathrm{~mm}$, the rest of others $17.4 \pm 9.2 \mathrm{~mm}, P<0.001$; size $>10 \mathrm{~mm}, P=0.025)$. However, rest of other factors including polyp location or morphology were not significantly associated with OD. In subgroup analysis in OD group, such as comparison between cases that were changed from neoplastic polyp to nonneoplastic lesion versus rest of others, none of the factors were significantly related. In subgroup analysis of cases that were changed from advanced adenoma to nonadvanced adenoma, significant factors were not found again. 
Table 6. Subgroup Analysis of Risk Factors according to Polyp Morphology

\begin{tabular}{|c|c|c|c|c|}
\hline \multirow{2}{*}{ Polyp morphology } & \multicolumn{2}{|c|}{ Histologic discordancy } & \multicolumn{2}{|c|}{ Under-diagnosis } \\
\hline & Adjusted OR $(95 \% \mathrm{Cl})$ & $P$-value & Adjusted OR $(95 \% \mathrm{Cl})$ & $P$-value \\
\hline Protruding ( $n=158)$ vs. flat $(n=132)$ & $1.350(0.848-2.148)$ & 0.206 & $1.356(0.811-2.267)$ & 0.244 \\
\hline Protruding ( $n=158$ ) vs. flat (except LST, $n=36$ ) & $4.249(1.758-10.268)$ & 0.001 & $3.913(1.310-11.690)$ & 0.010 \\
\hline Protruding ( $n=158)$ vs. LST ( $n=96)$ & $0.976(0.589-1.618)$ & 0.925 & $0.969(0.562-1.671)$ & 0.909 \\
\hline \multicolumn{5}{|l|}{ Size $\leq 10 \mathrm{~mm}$} \\
\hline Protruding ( $n=55)$ vs. flat $(n=36)$ & $1.983(0.695-5.659)$ & 0.196 & $0.887(0.254-3.300)$ & 1.000 \\
\hline \multicolumn{5}{|l|}{ Size $>10 \mathrm{~mm}$} \\
\hline Protruding ( $n=103)$ vs. LST (n=96) & $1.583(0.899-2.787)$ & 0.110 & $1.594(0.890-2.849)$ & 0.116 \\
\hline Lateral spreading tumors ( $n=96)$ & $1.337(0.823-2.186)$ & 0.245 & $1.181(0.693-2.012)$ & 0.540 \\
\hline Homogenous granular ( $n=31)$ & $0.804(0.341-1.893)$ & 0.617 & $0.544(0.202-1.461)$ & 0.223 \\
\hline Mixed nodular $(n=22)$ & $5.906(1.820-19.163)$ & 0.001 & $2.204(0.759-5.398)$ & 0.155 \\
\hline Flat elevated $(n=32)$ & $0.499(0.211-1.183)$ & 0.112 & $0.887(0.356-2.214)$ & 0.798 \\
\hline Pseudodepressed (n=11) & $0.484(0.132-1.778)$ & 0.267 & $1.185(0.319-4.399)$ & 0.799 \\
\hline
\end{tabular}

LST, lateral spreading tumor.

\section{DISCUSSION}

Polyps are generally classified as neoplastic or nonneoplastic polyps. ${ }^{20}$ Neoplastic polyps are adenomas which are benign epithelial tumors with variable malignant potential. ${ }^{21}$ The adenoma-carcinoma sequence is widely accepted, and it is known that more than $95 \%$ of CRC originate from adenomas. ${ }^{22}$ Inherited CRC syndromes such as familial polyposis and hereditary nonpolyposis CRC support such evidence and recent findings on ras gene mutation and chromosomal deletions additionally provide biological evidence of the adenoma-carcinoma sequence. ${ }^{3}$

In recent years, changes in the dietary lifestyle in the Korean population along with nationwide conducted cancer screening programs led to an increase in the prevalence of colorectal polyp and malignancy. ${ }^{23,24}$ Complete removal of adenomatous polyps is by far the most effective method to reduce the risk of CRC occurrence and subsequent cancerrelated death. ${ }^{25}$ However, complete polypectomy is not always attainable in actual clinical practice for several reasons. Possible complications of ER, such as bleeding, ${ }^{2}$ perforation, and postpolypectomy electrocoagulation syndrome, makes prompt total excision of colorectal polyp difficult, especially in primary medical institutions. Moreover, patients with coagulation disorders such as non-compensating cirrhosis or patients taking antiplatelet drugs or anticoagulants at the time of initial colonoscopy are not suitable candidates for one-step total excision of colorectal polyp. In such cases, EFB of the lesion followed by planned ER is usually performed.
However, indiscriminate EFB prior to polypectomy may lead to submucosal fibrosis and non-lifting of lesions, ${ }^{6}$ which makes the complete excision of the lesion more difficult. Therefore, careful biopsy for accurate histology with minimal effect on the procedure is critical. We performed this study to evaluate the value of EFB in accurately reflecting the actual nature of the totally resected colorectal polyp specimen, and to analyze the risk factors of HD, especially UD which is the most important pressing issue in clinical practice.

There have been several studies ${ }^{8-13}$ on HD between EFB and ER specimens in gastric neoplasm, with the HD rate ranging from $10 \%$ to approximately $50 \%$. In previously published reports of the stomach, significant predictors of HD include larger lesion size, depressed morphology, surface nodularity, presence of ulcer or ulcer scars within the lesion, or presence of erythema. However, the results are widely conflicting, thus complete histologic investigation of the ER specimen is essential for developing an optimal management plan.

Few studies have been conducted regarding HD between EFB and ER specimens in colon neoplasm, reporting a variable rate ranging between $10 \%$ and $40 \% .{ }^{14-18}$ Pugliese et al. ${ }^{17}$ published a study of 59 colorectal polyps which underwent repetitive EFB before ER, and reported that small polyps $(n=41)$ had $88 \%$ agreement between the histologic result of the EFB and ER specimen, but in large polyps ( $n=18)$, only $28 \%$ agreement was noted. Gondal et al. ${ }^{14}$ presented a histologic comparison of 532 colorectal polyps detected by flexible sigmoidoscopy screening that subsequently underwent 
colonoscopy for complete polypectomy. The HD and UD rate was about $10 \%$ and $7 \%$ respectively, and the diameter of the polyp was positively associated with an increased risk of UD at the time of EFB. In a similar study in Korea by Song et al., ${ }^{26}$ a histological investigation of 206 colorectal polyps in 137 patients was performed, and the HD rate was found to be $18.4 \%$. Polyps $>16 \mathrm{~mm}$ irrespective of morphology and polyps $\leq 10 \mathrm{~mm}$ with pedunculated morphology were more likely to be histologically discordant. On the other hand, most of the other studies failed to demonstrate significant predictors of HD. ${ }^{8,15,16}$

In our study, the HD and UD rates were $47.2 \%$ and $29.7 \%$, respectively. These rates are relatively higher than those reported in previous studies. The difference may be attributed to the nature of tertiary university medical center, as most of the cases in the present study were referred cases of relatively large sized polyps that could not be easily polypectomized in other hospitals.

Polyp size $>10 \mathrm{~mm}$ was a significant predictor of both HD and UD in the present study. In terms of polyp morphology, protruding or flat subtype was not significantly relevant. However, among LST subtypes, mixed nodular type showed an increasing tendency for HD when compared with other subtypes. It is a reasonable assumption that as the size of the polyp increases, the possibility of histopathological heterogeneity also increases, which explains why the findings of EFB of larger polyps and those of totally resected specimens tend to be discordant and malignant lesions could be underdiagnosed. Similarly, assuming the size is the same, protruding lesions have a larger volume of tissue when compared to flat subtypes, which may lead to HD by EFB. Considering that the main aim is to obtain a smaller biopsy specimen with highly accurate histologic diagnosis, biopsy should be performed carefully at the nodular portion so as not to miss the possibility of malignancy. Our study provides clear evidence of a relationship between polyp size and HD, and draw a conclusion that the polyp size is the single most important predictive factor of both HD and UD.

Interestingly, our study showed that polyp size was also associated with OD by EFB. It is well known that the distribution of histopathological features is not uniform in single adenoma. ${ }^{27}$ This may explain the case of OD as well as UD, as small foci of well differentiated adenocarcinoma may be removed by cold biopsy, leaving the bulk of the lesion with less severe histological features in totally resected specimens, as in the single case of OD observed in our study. In this case, we retrospectively reviewed the EFB specimen in our hospital again and the same pathologic assessment was made. In some respects, we can assume that OD cases are those that were relatively well-biopsied at critical portion within a polyp, especially in large polyp that has higher possibility of histopathological heterogeneity.

The present study has some limitations due to the retrospective nature of the study. We could only review the pathology reports from each local medical institutions, not the pathology specimens themselves. Since the pathologic assessment of EFB and ER specimens was made by different pathologists, it inevitably resulted in inter-observer variability. Assessing the degree of dysplasia of an adenoma as low or high is substantially subjective. There is no absolute definition of "high-grade" dysplasia. The same applies to assessing tubulovillous or villous adenoma as well as differentiation of adenocarcinoma. A study by Gondal et al. ${ }^{14}$ found that inter-pathologist variation is an independent predictor of HD between EFB and ER specimens, along with the polyp size. In our study, in only 5 cases, both EFB and ER were performed in our hospital, while in the rest of the cases, the patients were transferred from local medical centers after EFB and then underwent polypectomy in our hospital. This was associated with the nature of referral hospitals. Because of the enormous difference in the number of cases that underwent both procedures in our hospital and one procedure ( 5 vs. 285 , respectively), we could not include inter-observer variation as a statistical variable, although this might be a potential confounding factor that may affect the frequency of HD. However on the other side, in actual clinical practice, most patients come to tertiary hospital with just the pathology report, not with pathology specimen. We believe that our result may reflect the actual clinical situation in referral hospitals.

Besides, due to such nature of referral hospital, there might be a referral bias. Not all colorectal polyps found in the clinical settings are needed to be referred to tertiary hospital. Thus, the results of our study cannot be generalized to all colorectal polyps. Also, since each EFB were performed at different medical centers in retrospective setting, we could not standardize biopsy protocol. Among cases that were stated with specific number of EFB performed (198 cases out of 290 polyps), most of the EFB (96.5\%) were performed only once per polyp. However, the number of biopsy and the optimal location of biopsy within a polyp was not perfectly controlled. Thus, the absence of standardized biopsy protocol may have affect the rate of HD.

Despite these limitations, however, our study is the first to evaluate the rate of HD based on fully examined colonoscopic findings, and further analyze the risk factors of 
HD, UD and OD in a relatively larger number of subjects compared with those included in previous reports. HD was noted in $47.2 \%$ of colorectal neoplasm, and about $29.7 \%$ of polyps were underestimated and $14.5 \%$ of polyps were overestimated by EFB prior to complete polypectomy. Polyp size $>10 \mathrm{~mm}$ was the single most important predictive factor for HD, UD or OD. Careful biopsy for an accurate histological diagnosis before the procedure and choosing the optimal treatment strategy for complete resection of colorectal neoplasm is warranted, especially when the size of the polyp is $>10 \mathrm{~mm}$.

\section{FINANCIAL SUPPORT}

This work was supported by 2017 Yeungnam University Research Grant

\section{CONFLICT OF INTEREST}

No potential conflict of interest relevant to this article was reported.

\section{AUTHOR CONTRIBUTION}

M.J.H. wrote the paper; A.L.K acquired, analyzed and interpreted the data; S.H.L., B.I.J., T.N.K. assisted the data analysis, revised the manuscript; K.O.K. designed the study, revised the manuscript for critical intellectual content and approved the final version.

\section{REFERENCES}

1. El Zoghbi M, Cummings LC. New era of colorectal cancer screening. World J Gastrointest Endosc 2016;8:252-258.

2. Kwon MJ, Kim YS, Bae SI, et al. Risk factors for delayed postpolypectomy bleeding. Intest Res 2015;13:160-165.

3. Vogelstein B, Fearon ER, Hamilton SR, et al. Genetic alterations during colorectal-tumor development. N Engl J Med 1988;319: 525-532.

4. Lee JL, Cha JM, Lee HM, et al. Determining the optimal surveillance interval after a colonoscopic polypectomy for the Korean population? Intest Res 2017;15:109-117.

5. Kim KO. Surveillance colonoscopy after polypectomy: actual practice in Korea. Intest Res 2014;12:83-84.

6. Friedland S, Shelton A, Kothari S, Kochar R, Chen A, Banerjee S. Endoscopic management of nonlifting colon polyps. Diagn Ther Endosc 2013;2013:412936. doi: 10.1155/2013/412936.
7. Coda S, Thillainayagam AV. State of the art in advanced endoscopic imaging for the detection and evaluation of dysplasia and early cancer of the gastrointestinal tract. Clin Exp Gastroenterol 2014;7:133-150.

8. Sung HY, Cheung DY, Cho SH, et al. Polyps in the gastrointestinal tract: discrepancy between endoscopic forceps biopsies and resected specimens. Eur J Gastroenterol Hepatol 2009;21:190195.

9. Choi JM, Kim SG, Yang HJ, et al. Endoscopic predictors for undifferentiated histology in differentiated gastric neoplasms prior to endoscopic resection. Surg Endosc 2016;30:89-98.

10. Min BH, Kang KJ, Lee JH, et al. Endoscopic resection for undifferentiated early gastric cancer: focusing on histologic discrepancies between forceps biopsy-based and endoscopic resection specimen-based diagnosis. Dig Dis Sci 2014;59:2536-2543.

11. Shim CN, Kim H, Kim DW, et al. Clinicopathologic factors and outcomes of histologic discrepancy between differentiated and undifferentiated types after endoscopic resection of early gastric cancer. Surg Endosc 2014;28:2097-2105.

12. Takao M, Kakushima N, Takizawa K, et al. Discrepancies in histologic diagnoses of early gastric cancer between biopsy and endoscopic mucosal resection specimens. Gastric Cancer 2012;15:91-96.

13. Yoon WJ, Lee DH, Jung YJ, et al. Histologic characteristics of gastric polyps in Korea: emphasis on discrepancy between endoscopic forceps biopsy and endoscopic mucosal resection specimen. World J Gastroenterol 2006;12:4029-4032.

14. Gondal G, Grotmol T, Hofstad B, Bretthauer M, Eide TJ, Hoff G. Biopsy of colorectal polyps is not adequate for grading of neoplasia. Endoscopy 2005;37:1193-1197.

15. Stermer E, Bejar J, Miselevich I, et al. Do forceps biopsies truthfully reflect the nature of endoscopically uncovered polypoid lesions of the colon? Colorectal Dis 2005;7:345-349.

16. Livstone EM, Troncale FJ, Sheahan DG. Value of a single forceps biopsy of colonic polyps. Gastroenterology 1977;73:1296-1298.

17. Pugliese V, Gatteschi B, Aste H, et al. Value of multiple forceps biopsies in assessing the malignant potential of colonic polyps. Tumori 1981;67:57-62.

18. Taylor EW, Thompson H, Oates GD, Dorricott NJ, AlexanderWilliams J, Keighley MR. Limitations of biopsy in preoperative assessment of villous papilloma. Dis Colon Rectum 1981;24: 259-262.

19. ASGE Standards of Practice Committee, Fisher DA, Shergill $\mathrm{AK}$, et al. Role of endoscopy in the staging and management of colorectal cancer. Gastrointest Endosc 2013;78:8-12.

20. Su MY, Hsu CM, Lin CJ, et al. Endoscopic treatment of colorectal neoplasms: a simple and safe procedure to lower the incidence of colorectal cancers. Dig Dis Sci 2008;53:1297-1302. 
21. Winawer SJ, O'Brien MJ, Waye JD, et al. Risk and surveillance of individuals with colorectal polyps. Who Collaborating Centre for the Prevention of Colorectal Cancer. Bull World Health Organ 1990;68:789-795.

22. Bujanda L, Cosme A, Gil I, Arenas-Mirave JI. Malignant colorectal polyps. World J Gastroenterol 2010;16:3103-3111.

23. Park Y, Lee J, Oh JH, Shin A, Kim J. Dietary patterns and colorectal cancer risk in a Korean population: a case-control study. Medicine (Baltimore) 2016;95:e3759. doi: 10.1097/MD.0000000 000003759.
24. Jung YS, Park DI, Kim WH, et al. Risk of advanced colorectal neoplasia according to the number of high-risk findings at index colonoscopy: a Korean Association for the Study of Intestinal Disease (KASID) Study. Dig Dis Sci 2016;61:1661-1668.

25. Kwon SU, Lee EJ, Eun JR, et al. The significance of small polyp of colon in Koreans. Yeungnam Uni J Med 2000;17:39-48.

26. Song ID, Kim JW, Oh IS. The pathological differences of colorectal polyps examined between the use of a forcep biopsy and endoscopic resection. Korean J Gastrointest Endosc 2008;37:1419.

27. Fung $\mathrm{CH}$, Goldman $\mathrm{H}$. The incidence and significance of villous change in adenomatous polyps. Am J Clin Pathol 1970;53:2125. 